Research Article

\title{
Improving the Tracking Performance under Nonlinear Time-Varying Constraints in Motion Control Applications: From Theoretical Servo Model to Experimental Validation
}

\author{
Hung Nguyen, ${ }^{1}$ Thanh Phuong Nguyen, ${ }^{1}$ and Ha Quang Thinh Ngo $\mathbb{D D}^{2,3}$ \\ ${ }^{1}$ HUTECH Institute of Engineering, Ho Chi Minh City University of Technology (HUTECH), Ho Chi Minh City, Vietnam \\ ${ }^{2}$ Department of Mechatronics Engineering, Faculty of Mechanical Engineering, \\ Ho Chi Minh City University of Technology (HCMUT), 268 Ly Thuong Kiet Street, District 10, Ho Chi Minh City, Vietnam \\ ${ }^{3}$ Vietnam National University Ho Chi Minh City, Linh Trung Ward, Thu Duc District, Ho Chi Minh City, Vietnam
}

Correspondence should be addressed to Ha Quang Thinh Ngo; nhqthinh@hcmut.edu.vn

Received 1 April 2021; Accepted 12 June 2021; Published 22 June 2021

Academic Editor: M Syed Ali

Copyright ( $\odot 2021$ Hung Nguyen et al. This is an open access article distributed under the Creative Commons Attribution License, which permits unrestricted use, distribution, and reproduction in any medium, provided the original work is properly cited.

In the high-accuracy control of an AC machine, the knowledge of pure system parameters, with no deviation in drive coefficients and no disturbance or other nonlinear components, is a difficult issue for operators, even though it is occasionally nonviable. To overcome these troubles, this paper introduces a robust adaptation strategy based on pseudo fuzzy logic and sliding mode control (PFSMC) for an AC servo drive subject to uncertainties and/or external disturbance. Owing to the robustness of the SMC technique, the reduced sensitivity to uncertainties, and the enhanced resistance to disturbances from the pseudo fuzzy mechanism, this control algorithm can guarantee not only system stability but also the improvement of tracking errors in the steady state. To validate the design efficiency of PFSMC, both simulation and laboratory tests of the proposed scheme and a conventional PID scheme were performed to compare them as follows. In a computer environment, test cases with and without certainties were implemented with two controllers to visualize the comparative responses. Then, the two control methods were integrated into a real-world hardware platform to acquire practical outcomes. From these results, it can be noted that our successful approach showed a feasible, effective, and robust performance in AC drive.

\section{Introduction}

At present, the demands in high-precision machines have been sharply increased to correlate with numerous fields (manufacturing tools, aerospace, innovative robotics system, etc.). Servo mechanisms as well as servo actuators are indispensable components in advanced machines. The enlargement of servo systems would inspire the growth of other industries. Hence, many developers have focused on them, from a vibration-assisted milling machine [1], a hydraulic actuator coupled with a nonlinear physical system [2], and mechanical parameters in a two-mass servo drive system [3] to a duo-servo vehicle brake [4] or 3D pose tracking for underwater vehicles [5]. An integrated size and a lightweight motor are desired for high-performance applications. Consequently, the volume and dimensions may be important factors in medical robots. Depending on each industrial field, the technical specifications, electrical features, and dynamic characteristics may differ.

A servo pneumatic system has a widespread range of applications in industrial automation, such as packaging, supervising, assembly, or clamping. This technique is significantly helpful for physical system manipulation, the fast movement of linear components, safe and clean technology, low cost and low maintenance, high mechanical efficiency and long working life [6, 7]. Nonlinear characteristics generally have a negative effect on the precision of the overall system. The nonlinear frictional forces, the thermodynamics of the air flow in the cylinder, the varying load, the uncertain features of valve actuators, and the unprofitable energy are 
all limitations of servo pneumatic actuator systems. The control problems that most research focuses on are the response of exactness and velocity of individual actuators. The step change in reference input could lead to tests of position modification or point to point.

The considerable developments in the current era have made an impact on various technologies of robot and automation systems close to each other. In the general industrial sector [8], automation relies on electric, mechanic, and information technology components to handle all processes. Specifically, if robotics is the main element of an automated system, then motion control techniques are the core of the robot system. Based on the servoing motion knowledge, an industrial robot could be manipulated for multifunctional missions, and its movement could be reprogrammed to carry materials, parts, tools, or devices through continuously varying motion. Currently, it is considered to be an implementing component of industries due to its performance in enhancing precision, repeatability, reliability, and flexible drive. As key factors in economic progress, industrial manipulators are replacing humans in harsh environments to perform tedious tasks.

Servo drives are commonly utilized in high-tech motion systems; AC servo motors in particular have brought many great benefits, such as high speed, better efficiency, small steady-state error, and low noise. Along with significant success in the semiconductor industry, many efforts have been expended to research the AC drive in order to yield commercial products in the global market. In the new trend, it is compulsory to combine the driving motor with an inverter and its controller. This kind of motor offers a speed near nominal values with the power supply frequency. Conversely, the ultra-high-velocity drive technique of a large-capacity motor diverges with high-velocity drive in the rotor surface, magnetic bearing, material, and assembly routine [9].

\section{Background Work}

From the above-mentioned applications, successful developments in robotics systems are obviously related to proper control laws in servo actuators. The primary objective of most of these implementations is to measure the corrective control signals to compensate for the many variations from different sources. The appropriate design helps to lessen the effects of external disturbances and causes the steady-state error to tend to zero whenever the system tracks precisely and nearly close to the target position [9]. The most popular scheme is the Proportional-Integral-Derivative (PID) control, significantly characterizing both industry and research. The challenge in this controller is to tune right gains either manually or automatically [11]. These methods consist of the open/closed-loop Ziegler-Nichols method, the integral of time-weighted absolute error method, and the Cohen-Coon method [12]. The advanced tuning procedures are used to integrate with fuzzy, neural network, adaptive control or others to adapt with fluctuating in the system. Other types of PID scheme include fractional-order and event-based structures, which are typical for a generalization of standard integer-order and wireless transmitters and actuators, respectively. Together with the required industrial applications, different modern control strategies exist, such as computed torque control [13, 14], sliding mode control [15], observer-based control [16], time-delayed control [17], and LQR [18]. Of these, the sliding mode control design reveals superior advantages as it shows robustness and proved stability in robotics systems. Modeling of servo actuators is often carried out to clarify the driving torque in motors. Although there are uncertainties in modeled parameters or external disturbances, the system can switch from one phase to another depending upon the current system's state.

In a high-precision $\mathrm{AC}$ machine, the complex integrations of numerous components involve the information of system parameters. Despite testing and validation efforts, knowledge of machinery data can be hard to identify exactly. As it cannot be determined occasionally, the identification procedure is a challenge in automation control [19]. As a result, machinery data estimation is considered a notable contribution in the development of AC drives. These features enable the electric drives to govern the machine parameters and tune the control loop. Based on the working principles, many methods are clustered in several categories of numerical methods. They rely on the modeling of finiteelement machines [20] and are only available for motor developers. Regular users who lack design data cannot successfully build an exact model of the machine. In the dynamo method [21], the dedicated instrumentation is set up as a speed-controlled follower and a driver. They are connected together as twin motors, and a host computer plays the role of data logger. The driving mechanism with different input signals is activated and the observed response is obtained through the measurement of electrical variables (voltage, current, and power). With time, the recorded data are obtained along with environmental variables (temperature and magnetic and electromagnetic fields). With this information, a synthesizing step is performed to form a flux mapping. The parameter identification process is accurately predictable to enhance the positioning control. In the other method, standstill determination, which is partly sectioned into frequency domain [22] and time domain [23], is simpler and needs rotor locking. In this way, the testing machine is delivered in a single-phase grid with various signal patterns to approximate the system's response. The setting facilities that are proper for offline techniques include a signal generator, high-resolution measuring instrument, and data recorder. However, without good instrumentation, some fundamental hardware is also able to accomplish accurate characterization of an AC drive. The self-commissioning technique [24] is a key classification system in modern drive systems. The characteristics of this alternative current drive are that it is built on a low-cost microcontroller, employs powerful computation using accessible sensors, does not need rotor locking and sources of driving torque, and requires the least operator intervention. The parameters of motorizing circuits can be simulated from steady-state measurements and standstill frequency responses by dissimilar excitation signals through a power inverter. Most modern commercial drives, in the initial routine, generate 
algorithms to identify the motor's parameters. Depending on the drive manufacturer, the identification process via the inverter may vary separately, but the main idea still depends on hardware resources and the control strategy. Consequently, the overview of research topics on servo systems is summarized in Figure 1, in which these approaches are grouped into various categories based on their operating principles.

2.1. Components of a Servo System. The main components of a servo system include the driving actuator, sensing device, control mechanism, and data acquisition system. Some industrial motor drives are commonly utilized in different applications, such as Permanent Magnet Synchronous Motor (PMSM) and Brushless DC (BLDC) [25], which have many similarities but fundamental differences in Electromotive Force (EMF). Recently, a piezoelectric motor [26] driven by the change in shape of the piezoelectric material when an electric field is applied appeared in a highly precise control aspect. Furthermore, several articles on the advanced functions [27] or more complex structure [28] of piezo motors to optimize energy harvesting and to guarantee stable motion have been proposed.

Sensors play an important role in most industrial experiments because, if faulty, they may lead to failures in the drive system. Regularly, a linear incremental position encoder [29] is deployed for the accurate measurement of the displacement of a driving motor. Additionally, a linear potentiometer [30] coupled with the rod end of a lead-screw mechanism has also been utilized as a position transducer to evaluate the displacement. The accuracy, resolution, and data acquisition rate have significant impacts on the dynamics of a closed-loop system.

2.2. Modeling of a Servo System. Compared to regular motors, a servo system is specifically launched with consideration for the moment of inertia of the rotor and the electrical responses in such a way that it can react to sudden alterations of voltage and current from the servo amplifier. Initially, the mathematical model of a servo drive can be illustrated by fourth-order equations [31]. In some cases, advanced control algorithms $[32,33]$ are studied in order to achieve high performance. If the friction force is considered, then a compensator $[34,35]$ should be added to reject unexpected disturbances.

2.3. Tracking Control of a Servo System. In the field of control, one of the main research objects is the whole servo system, which usually uses both linear and nonlinear schemes. Each control method has its own advantages-for example, feedback linearization [36] and LQR [37] reveal a superior performance in optimal parameters. For the class of nonlinear control, several studies have been carried out-for instance, tracking control using asymptotic prescribed performance [38] and indirect adaptive control [39]. In recent years, intelligent algorithms including fuzzy [40] or neural networks [41] have been introduced into complex servo systems to boost their performance. Furthermore, to improve the tracking control in finite time, the nonsingular fast terminal sliding mode strategy has been used to deliver a rapid response and control precision via an extended state observer [42].

2.4. Motion Profile of a Servo System. For a large number of applications, including medical automation, scientific instrumentation, and many types of general automation, point-to-point movement is employed more frequently than other profiles. One of the most popular motion profiles for a point-to-point approach is the S-curve or fourth-order symmetric polynomial [43]. For a large number of industrial applications, exponential trajectory planning [44] and trigonometric-function-based generators [45] are options to drive the servo system to track its desired path.

2.5. Vibrationless Control of a Servo System. The real-world applications of the above profiles aim to achieve fast movement with low vibration $[46,47]$ and nanopositioning measurement [48]. As for other aspects, smoothness and the time-optimal effect are also valuable for automated robots and machines [49]. From the viewpoint of filtering performance, some studies of applications in FIR (finite impulse response) [50] or exponential filter [51] have been conducted. Another research study focused on jerk-continuous profile in order to generate a smooth trajectory for robotic manipulators [52].

2.6. Identification Parameters of a Servo System. Numerical approaches rely on finite element modeling of electrical machines [53]. The disadvantage of this method is that it is only accessible for motor designers, since others do not have enough data to establish a numerical or finite element model of the machine. In another approach, dynamo [21] requires a speed-controlled prime mover with dedicated instrumentation. It maps the flux of a machine and utilizes phasor interpretation to obtain equivalent circuit parameters. Furthermore, the standstill identification approach [54] is one of the simpler applications and usually needs rotor locking. It could be classified into a frequency response and time response based on the principle of parameter estimation utilized. In both cases, the automated machine has a single phase during the test with various signal patterns to evaluate the system parameters according to the response. Last but not least, the parameter identification techniques that require the rotor shaft to be free to rotate are categorized individually under the heading of free shaft [55].

\section{Mathematical Model of a Servo System}

The dynamics equation of an AC servo system is described in

$$
J \ddot{\theta}+B \dot{\theta}+\tau_{d}=\tau,
$$

where $\theta$ is angular movement of motor; $J$ is inertial moment of motor; $B$ is viscous coefficient; $\tau$ is driven torque; $\tau_{d}$ is 


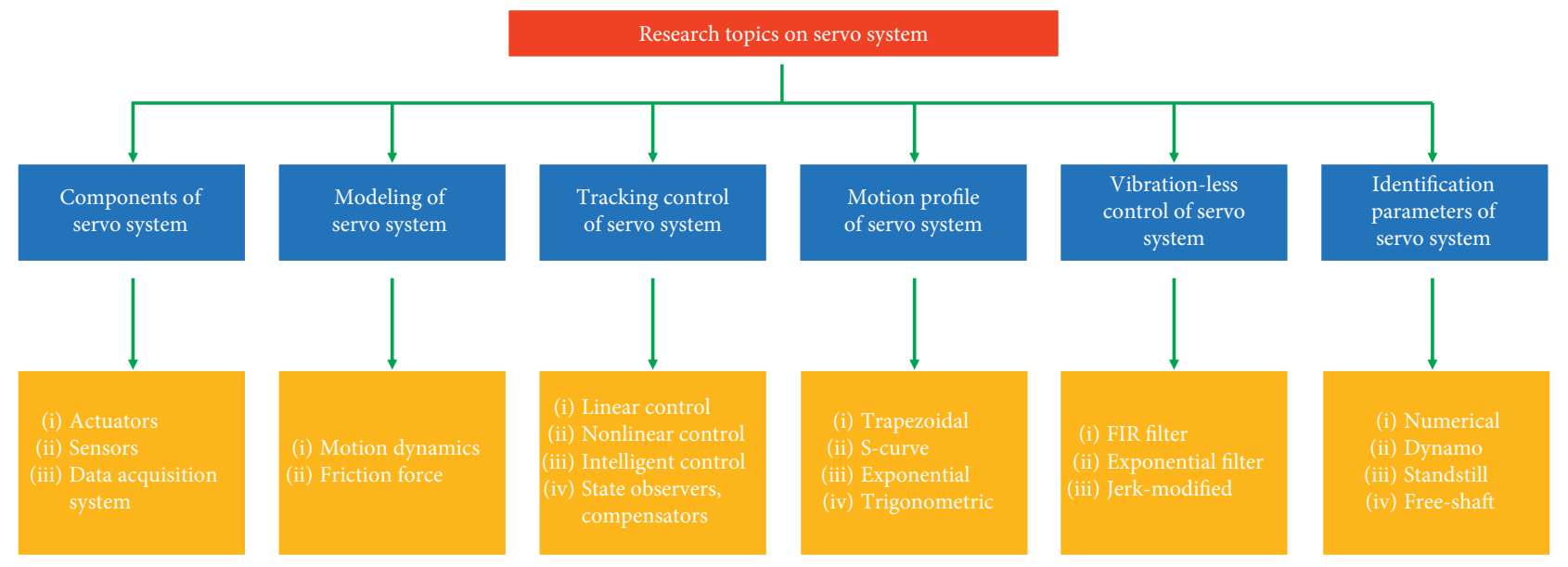

Figure 1: Summary of related studies on servo systems.

external disturbance torque from unknown load on rotational motor shaft.

Equation (1) could be rewritten in state space form:

$$
\left[\begin{array}{l}
\dot{e}_{1} \\
\dot{e}_{2}
\end{array}\right]=\left[\begin{array}{cc}
0 & 1 \\
-a_{1} & -a_{2}
\end{array}\right]\left[\begin{array}{l}
e_{1} \\
e_{2}
\end{array}\right]+\left[\begin{array}{l}
0 \\
b
\end{array}\right]\left(\tau-\tau_{r}\right) \Leftrightarrow \dot{x}=A x+B u+w,
$$

where $e_{1}=\theta-\theta_{r} ; e_{2}=\dot{\theta}-\dot{\theta}_{r} ; \tau_{r}=J \ddot{\theta}+B \dot{\theta}_{r}+\tau_{d} ; \theta_{r}$ is reference angular movement of motor; $x=\left[\begin{array}{l}e_{1} \\ e_{2}\end{array}\right], a_{1}=0, a_{2}=(B / J), b=-(1 / J) ;$ and $\Delta a_{i}$ and $\Delta b_{i}$, $i=1,2$ are uncertainties in the servo system. The following assumptions are made.

Assumption 1. The uncertainties $\Delta a_{i}$ and $\Delta b_{i}, i=1,2$ are continuously functions of a vector of uncertain constraints $p \in P \subset \mathfrak{R}^{p}$

$$
\begin{aligned}
\Delta a_{i} & =\Delta a_{i}(p), \\
\Delta b_{i} & =\Delta b_{i}(p) .
\end{aligned}
$$

Assumption 2. There exist unknown positive bounds $\delta_{i}$ and $\varepsilon_{i}, i=1,2$ and $v(x, t)$, such that

$$
\begin{aligned}
a & =\widehat{a}+\Delta a=\widehat{a}+\widehat{b} D(p), \\
b & =\widehat{b}+\Delta b=\widehat{b}+\widehat{b} E(p), \\
w(x, t) & =\widehat{b} v(x, t) .
\end{aligned}
$$

The boundary conditions are satisfied as follows:

$$
\begin{aligned}
\left|D_{i}(p)\right| \leq \delta_{i}, & i=1,2, \\
\left|E_{i}(p)\right| \leq \varepsilon_{i}, & i=1,2, \\
\left|v_{i}(x, t)\right| \leq v_{i}, & \forall x, \forall t, i=1,2 .
\end{aligned}
$$

The uncertainties $\Delta a_{i}$ and $\Delta b_{i}, i=1,2$ denote variations in the system parameters and impact from bounded external perturbations. The upper bounds $\delta_{i}$ and $\varepsilon_{i}$ ensure that the varying effect does not cause disturbances such that it would reverse the control action.

\section{Proposed Approach}

Sliding control is the constrained motion of states along trajectories on the sliding surface $s$ that is chosen as follows:

$$
s=C x=\left[\begin{array}{ll}
c_{1} & 1
\end{array}\right]\left[\begin{array}{l}
e_{1} \\
e_{2}
\end{array}\right]=e_{2}+c_{1} e_{1} .
$$

The coefficient $c_{1}$ is symbolized by $\left\{\lambda \mid \lambda+c_{1}=0\right\}$ from the sliding surface.

By selecting the Lyapunov candidate function

$$
V=\frac{1}{2} s^{T} s
$$

the condition to stabilize in a servo system is

$$
\dot{V}=s^{T} \dot{s}<0 .
$$

To satisfy the above condition, the components of motion controller should be taken as

$$
u=u_{\mathrm{Eq}}+u_{\mathrm{Sl}}+u_{\mathrm{Fz}}
$$

where $u_{\mathrm{Eq}}$ is the equivalent state of the controller; $u_{\mathrm{Sl}}$ is the sliding state of the controller; and $u_{\mathrm{Fz}}$ is the fuzzy state of the controller. follows.

The components of the controller can be found as

4.1. Equivalent State. The essential situation for the state path to maintain on the sliding surface $s$ is $\dot{s}=0$. In this perfect case, the nominal system containing $\Delta a_{i}=0, \Delta b_{i}=0$ stays on the sliding surface $s$ if

$$
\left[\begin{array}{ll}
c_{1} & 1
\end{array}\right]\left(\left[\begin{array}{cc}
0 & 1 \\
-a_{1} & -a_{2}
\end{array}\right]\left[\begin{array}{l}
e_{1} \\
e_{2}
\end{array}\right]+\left[\begin{array}{l}
0 \\
b
\end{array}\right] u_{\mathrm{Eq}}\right)=0 .
$$

For a closed-loop system, $u_{\mathrm{Eg}}$ is assigned from the desired dynamics of the linear feedback system 


$$
u_{\mathrm{Eq}}=-K_{\mathrm{Eq}}^{0} x .
$$

Substituting (11) into (10), $K_{\mathrm{Eq}}^{0}$ is determined as

$$
K_{\mathrm{Eq}}^{0}=\left(\left[\begin{array}{ll}
c_{1} & 1
\end{array}\right]\left[\begin{array}{l}
0 \\
b
\end{array}\right]\right)^{-1}\left[\begin{array}{ll}
c_{1} & 1
\end{array}\right]\left[\begin{array}{cc}
0 & 1 \\
-a_{1} & -a_{2}
\end{array}\right]=-J\left[\begin{array}{ll}
0 & c_{1}-\frac{B}{J}
\end{array}\right] .
$$

Equation (11) is expressed as

$$
u_{\mathrm{Eq}}=J\left(\frac{B}{J}-c_{1}\right) e_{2} \text {. }
$$

4.2. Switching State. If the servo works in this case, the system state $x$ may not be located on the sliding surface $s$ at the initial stage, or it leaves from surface $s$ due to the effect of varying parameters or external disturbances. The motion controller must perform driving action of system state $x$ back to sliding mode $s=0$.

Theorem 1. Assume that dynamics system (1) with nonlinear characteristics satisfying Assumptions 1 and 2. If the sliding mode control is formed as

$$
u=u_{\mathrm{Eq}}+u_{\mathrm{Sl}}=-K_{\mathrm{Eq}}^{0} x+u_{\mathrm{Sl}},
$$

with equivalent control gain $K_{E q}^{0}$ given in (12) and sliding control $u_{S l}$

$$
K_{\mathrm{Sl}, i}=\frac{\left(\delta_{i}+\varepsilon_{i}\left|K_{\mathrm{Eq}, i}^{0}\right|\right)}{1-\varepsilon_{i}} \operatorname{sgn}\left(s^{T} C b_{i} x_{i}\right),
$$

and the sliding control signal is

$$
u_{\mathrm{Sl}}=-K_{\mathrm{Sl}} x
$$

Then, the system state $x$ asymptotically converges to zero.

Proof. Using the positive definite Lyapunov function (7), we have

$$
\begin{aligned}
\dot{V} & =s^{T} \dot{s}=s^{T} C[(\hat{a}+\Delta a) x+(\hat{b}+\Delta b) u+w] \\
& =s^{T} C\left[(\hat{a}+\Delta a) x+(\hat{b}+\Delta b)\left(-K_{\mathrm{Eq}}^{0} x+u_{\mathrm{Sl}}\right)+w\right] .
\end{aligned}
$$

From (10), $\dot{V}$ can be obtained:

$$
\begin{aligned}
\dot{V} & =s^{T} C\left[\Delta a x-\Delta b K_{\mathrm{Eq}}^{0} x+b u_{\mathrm{Sl}}+\Delta b u_{\mathrm{Sl}}+w\right]=s^{T} \hat{C b}\left[\left(D-E K_{\mathrm{Eq}}^{0}\right) x+(I+E) u_{\mathrm{Sl}}+v\right] \\
& =\sum_{i=1}^{2} \Theta_{j}\left[\left(D_{i j}-E_{j j} K_{\mathrm{Eq}, i j}^{0}\right) x+\left(1+E_{i j}\right) u_{\mathrm{Sl}, j}+v_{j}\right]=\sum_{i=1}^{2} \Theta_{j}\left[f_{j}^{T} x+\left(1+E_{i j}\right) u_{\mathrm{Sl}, j}+v_{j}\right],
\end{aligned}
$$

where $I$ is an identity matrix $2 \times 2$ and $f_{j}^{T}$ is the $j$ th row of the matrix $D-E K_{E}$.

Then, there are two possible cases for $\varphi_{j}(x)$. In the case that $\varphi_{j}(x)>0$, we have

$$
\begin{aligned}
\dot{V}<0 & \Longleftrightarrow \sum_{i=1}^{2}\left(D_{i j}-E_{j j} K_{\mathrm{Eq}, j i}^{0}\right) x_{i}+\left(1+E_{j j}\right) u_{\mathrm{Sl}, j}+v_{j}<0 \\
& \Longleftrightarrow u_{\mathrm{Sl}, j}<\frac{v_{j}+\sum_{i=1}^{2}\left(D_{i j}-E_{j j} K_{\mathrm{Eq}, j i}^{0}\right) x_{i}}{1+E_{j j}} .
\end{aligned}
$$

Therefore, the control signal should be designed as

$$
u_{\mathrm{Sl}, j}=-\frac{v_{j}+\left(\sum_{i=1}^{2}\left(\delta_{i}+\varepsilon\left|K_{\mathrm{Eq}, j i}^{0}\right|\right) x_{i}\right)}{1-\varepsilon} .
$$

Otherwise, $\varphi_{j}(x)<0$ :

$$
\begin{aligned}
\dot{V}<0 & \Longleftrightarrow \sum_{i=1}^{2}\left(D_{i j}-E_{j j} K_{\mathrm{Eq}, j i}^{0}\right) x_{i}+\left(1+E_{j j}\right) u_{\mathrm{Sl}, j}+v_{j}>0 \\
& \Longleftrightarrow u_{\mathrm{Sl}, j}>\frac{v_{j}+\sum_{i=1}^{2}\left(D_{i j}-E_{j j} K_{\mathrm{Eq}, j i}^{0}\right) x_{i}}{1+E_{j j}} .
\end{aligned}
$$

To meet the condition, the output of the controller is

$$
u_{\mathrm{Sl}, j}=-\frac{v_{j}+\left(\sum_{i=1}^{2}\left(\delta_{i}-\varepsilon\left|K_{\mathrm{Eq}, j i}^{0}\right|\right) x_{i}\right)}{1-\varepsilon} .
$$

From (20) and (22), the sliding control can be identified as follows:

$$
u_{\mathrm{Sl}, j}=-\frac{v_{j}+\left(\sum_{i=1}^{2}\left(\delta_{i}+\varepsilon\left|K_{\mathrm{Eq}, j i}^{0}\right|\right) x_{i}\right)}{1-\varepsilon} \operatorname{sgn}\left(\varphi_{j} x_{i}\right) .
$$

Consequently, the Lyapunov candidate function is ensured to be less than or equal to zero. 
4.3. Fuzzy Control. A novel fuzzy scheme is proposed to implement into the controller to enhance the overall performance. We consider that the fuzzy control signal $u_{\mathrm{Fz}, i}$ is expressed as

$$
u_{\mathrm{Fz}, i}=-\gamma_{i} \varphi_{i}(x), \quad i=1,2,
$$

where $\gamma_{i}$ is the output of the fuzzy scheme.

The fuzzy signal $u_{\mathrm{Fz}, i}$ must comply with fuzzy rules, which depend on variations of $s_{i}$ and $\Delta s_{i}$. In sliding mode control, there are oscillations in the system's state when the state trajectory would tend toward a sliding surface rapidly. If the system state is far from the surface, then $\left|s_{i}\right|$ becomes larger. As a result, the sliding coefficients increase correspondingly. If the state deviates from plane $\left(s_{i} \Delta s_{i}>0\right)$ and $\left|\Delta s_{i}\right|$ is greater, the coefficients of the system must drive the state back. On the other hand, if the state trajectory moves toward the sliding plane closely $\left(s_{i} \Delta s_{i}<0\right)$ and $\left|\Delta s_{i}\right|$ is bigger, the sliding parameters would decline to suppress oscillations. For the above purposes, the fuzzing processes $\left|s_{i}\right|$ and $\left|\Delta s_{i}\right|$ in two fuzzy linguistic variables large and smallare defined as follows:

$$
\begin{aligned}
& \mu_{s_{i}}^{\text {large }}=1-e^{\left(-\left(\left|s_{i}\right| / \eta_{s_{i}}\right)\right),} \\
& \mu_{s_{i}}^{\text {small }}=e^{\left(-\left(\left|s_{i}\right| / \eta_{s_{i}}\right)\right)}, \\
& \mu_{\Delta s_{i}}^{\text {large }}=1-e^{\left(-\left(\left|\Delta s_{i}\right| / \eta_{\Delta s_{i}}\right)\right),} \\
& \mu_{\Delta s_{i}}^{\text {small }}=e^{\left(-\left(\left|\Delta s_{i}\right| / \eta_{\Delta s_{i}}\right)\right),}
\end{aligned}
$$

where $\eta_{s_{i}}$ and $\eta_{\Delta s_{i}}$ are tuning coefficients positively.

The fuzzy rules are described briefly in Table 1 .

Since it is necessary to maintain the real-time performance of the servo system, singleton membership functions of fuzzy output are selected instead of triangular or trapezoidal shapes.

$$
\begin{aligned}
& \mu_{\gamma_{i}}^{\text {small }}= \begin{cases}1, & \gamma_{i}=0, \\
0, & \gamma_{i} \neq 0,\end{cases} \\
& \mu_{\gamma_{i}}^{\text {large }}= \begin{cases}1, & \gamma_{i}=\gamma_{i}^{m}, \\
0, & \gamma_{i} \neq \gamma_{i}^{m},\end{cases}
\end{aligned}
$$

TABLE 1: Table of fuzzy tuning rules.

\begin{tabular}{lccc}
\hline Rule no. & If & And & Then \\
\hline 1 & $\left|s_{i}\right|$ is large & & $\gamma_{i}$ is large \\
2 & $\left|s_{i}\right|$ is small & & $\gamma_{i}$ is large \\
3 & $s_{i} \Delta s_{i}>0$ & $\left|\Delta s_{i}\right|$ is large & $\gamma_{i}$ is large \\
4 & $s_{i} \Delta s_{i}>0$ & $\left|\Delta s_{i}\right|$ is small & $\gamma_{i}$ is small \\
5 & $s_{i} \Delta s_{i}<0$ & $\left|\Delta s_{i}\right|$ is large & $\gamma_{i}$ is small \\
6 & $s_{i} \Delta s_{i}<0$ & $\left|\Delta s_{i}\right|$ is small & $\gamma_{i}$ is large \\
\hline
\end{tabular}

where $\gamma_{i}^{m}$ is any proportional nonnegative constant.

To defuzzify, we could form the output by using the maxmin method.

$$
\gamma_{i}= \begin{cases}\gamma_{i}^{m}\left(1-e^{\left(-\left(\left|s_{i}\right| / \eta_{s_{i}}\right)\right)}\right)\left(1-e^{\left(-\left(\left|\Delta s_{i}\right| / \eta_{\Delta s_{i}}\right)\right)}\right), & s_{i} \Delta s_{i}>0, \\ \gamma_{i}^{m}\left(1-e^{\left(-\left(\left|s_{i}\right| / \eta_{s_{i}}\right)\right)}\right) e^{\left(-\left(\left|\Delta s_{i}\right| / \eta_{\Delta s_{i}}\right)\right)}, & s_{i} \Delta s_{i}<0 .\end{cases}
$$

Theorem 2. Assume that dynamics system (1) with nonlinear characteristics satisfies Assumptions 1 and 2. The output controller in Figure 2 is formed as

$$
u=u_{\mathrm{Eq}}+u_{\mathrm{Sl}}+u_{\mathrm{Fz}}
$$

where the equivalent control signal $u_{E q}$ is given in (11), the sliding switching control signal $u_{S l}$ is defined in (16), and the fuzzy control signal $u_{F z}$ is identified in (24). Then, the system state $x(t)$ asymptotically converges to zero.

Proof. Using the Lyapunov function candidate in (7) and the controller signal in (28), the 1 st derivative of $V(t)$ is presently demonstrated as

$$
\begin{aligned}
\dot{V} & =s^{T} \dot{s}=s^{T} C B\left[\left(D+E K_{\mathrm{Eq}}^{0}\right) x+(I+E)\left(u_{\mathrm{Sl}}+u_{\mathrm{Fz}}\right)+v\right] \\
& =\sum_{j=1}^{2} \varphi_{j}\left[f_{j}^{T} x+\left(1+E_{i j}\right)\left(u_{\mathrm{Sl}, j}+u_{\mathrm{Fz}}\right)+v_{j}\right]=\varphi\left[f^{T} x+(1+E)\left(u_{\mathrm{Sl}}+u_{\mathrm{Fz}}\right)+v\right],
\end{aligned}
$$

with $\varphi \neq 0$; condition (8) could be satisfied if, and only if,

$$
\varphi\left[f^{T} x+(1+E)\left(u_{\mathrm{Sl}}+u_{\mathrm{Fz}}\right)+\nu\right]<0 .
$$

If the fuzzy control signal is determined as (24), then

$$
\dot{V}=\varphi\left[f^{T} x+(1+E) u_{\mathrm{Sl}}+\nu\right]-(1+E) \gamma \varphi^{2}
$$

It is known that $\varphi\left[f^{T} x+(1+E) u_{\mathrm{Sl}}+\nu\right]<0$ (from Theorem 1$),(1+E)>0$ and $\gamma>0$. Hence, the sliding mode controller with the added fuzzy component can be expressed as

$$
\dot{V}=\varphi\left[f^{T} x+(1+E) u_{\mathrm{Sl}}+\nu\right]-(1+E) \varphi u_{\mathrm{Fz}}^{0} \tanh \left(\frac{\varphi}{\sigma_{\varphi}}\right)<0 .
$$




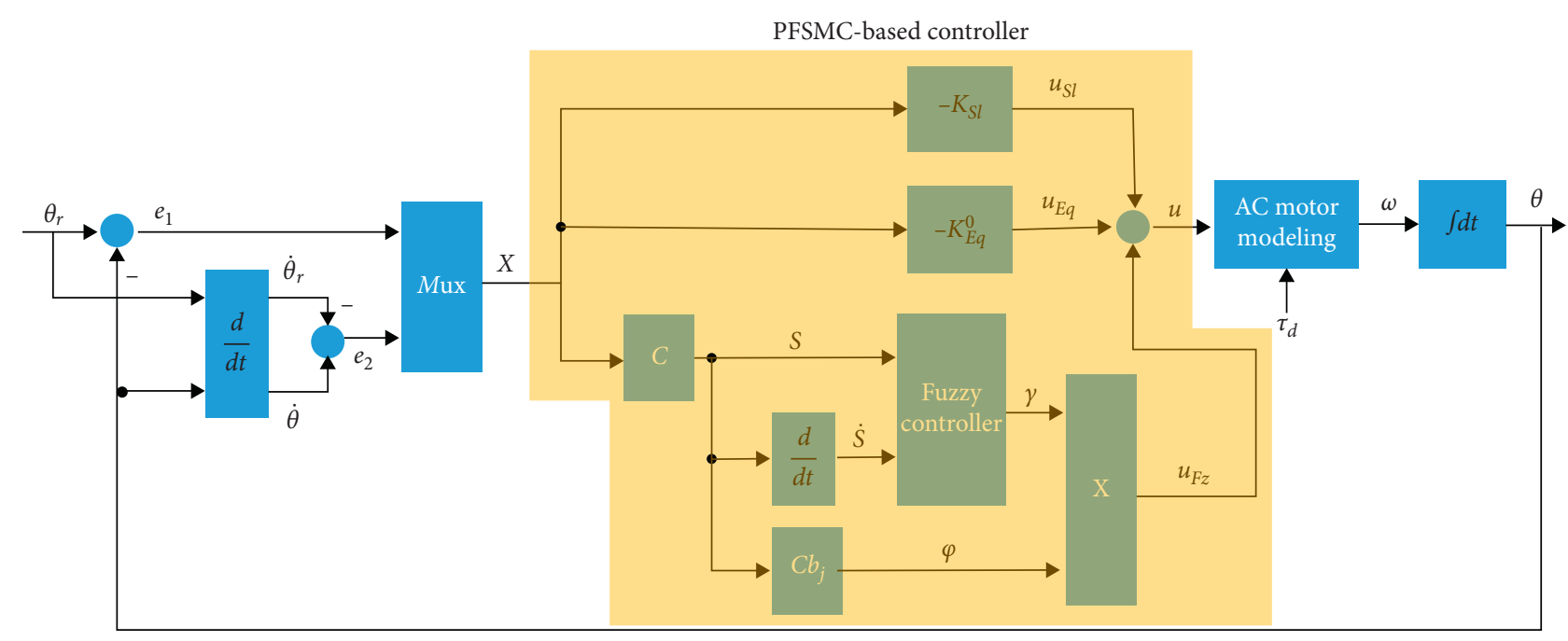

FIGURE 2: Block diagram of proposed PFSMC-based controller for a servo system.

It can be seen that proposed controller (27) suppresses the approximation tracking error and uncertainties, permitting the positioning error to converge to zero asymptotically due to the Lyapunov principle.

\section{Results of Numerical Simulation}

By presenting the pseudo-fuzzy law with the robust sliding mode control, the effectiveness of proposed scheme was firstly validated by numerical simulation. The target AC motor and driver were from Higen Motor [56], which have an industrial performance and are certified by international accreditation. The servo driver was operated in position control mode, which was measured by a 20-bit rotary encoder. The driving specifications were chosen as $J=1,6 \times 10^{-4} \mathrm{kgm}^{2}, \quad B=10^{-3}(\mathrm{Nm} / \mathrm{rad} / \mathrm{s}), \quad \varepsilon=0.1$, and $c_{1}=0.001$. In the pseudo-fuzzy structure, the selected parameters were considered as $\eta_{S_{i}}=\eta_{\Delta S_{i}}=100$ and $\gamma_{i}^{m}=25$. As demonstrated previously, the nonlinear servo system model was chosen to verify its applicability in practical industry. For that reason, the simulation tests were classified into two types: with and without uncertainties. Currently, the most common controller from servo manufacturers is the PID scheme, attracting many practitioners due to its advantages. The coefficients of the PID controller are given by $K_{P}=0.02 ; K_{I}=0.005 ; K_{D}=0.0015$. The comparative simulation results from a square pulse as the reference signal are provided to visualize the superior performance of proposed control strategy. In Figure 3, the position, angular velocity, and torque response of a conventional PID scheme without nonlinear servo characteristics are shown, as simulated on a computer. The blue line represents the reference input signal while the red line symbolizes the output response of the servo system. As expected, the PID tracking performance seems to struggle to follow the reference data when the input changes suddenly. It requires the operator's experience to tune the acceptable control parameters. The overshoot in tracking position geometrically seems to be large in the transient period. The PFSMC controller was implemented in the same configuration, and its results are demonstrated in Figure 4. Whenever the input signal changed, a nonzero tracking error appeared in the system state. The overall output of the control scheme adjusted the driving status to react in accordance with the fluctuation in the reference signal. The effectiveness of the proposed controller is revealed in the small overshoot and fast rising time.

In reality, nonlinear components or external disturbances will always exist in the driving model of an AC servo motor. They induce more difficulties in the motor control method. To imitate these situations, elements of uncertainties, which were simulated in a numerical computer, were added in the dynamic equation. The computer-generated performance with uncertainties in the PID strategy and in the proposed strategy is shown in Figures 5 and 6, respectively. Based on the rapid change of the input value, the output control responds to alter the feedback value. However, the Proportional-Integral-Derivative structure seemed to hardly adapt with uncertainties. As it cannot predict the nonlinear influences in the servo model, the response of the PID control oscillates, even though the system stays in a steady state. Unlike the conventional PID controller, the PFSMC could accommodate the internal variations in the AC servo motor. It is thought that the cause of nonlinear characteristics is due to the alternatives of PFSMC and setting of the modifications rule again. The simulation results in the uncertain model confirmed that vibration in the control signal still occurs, but with a lesser amplitude, and the PFSMC attempts to drive the angular error to converge to zero immediately. The comparison of the simulation results between PID and PFSMC is listed in Table 2. To deliver a general view, we compared their performances in three categories: overshoot, rising time, and tracking error. In each comparative item, we obtained results in a perfect case (without certain elements) and an imperfect case (with certain elements). It can be seen clearly that the proposed PFSMC shows a better performance, with a fast response, low tracking error, and adaptive characteristics. 


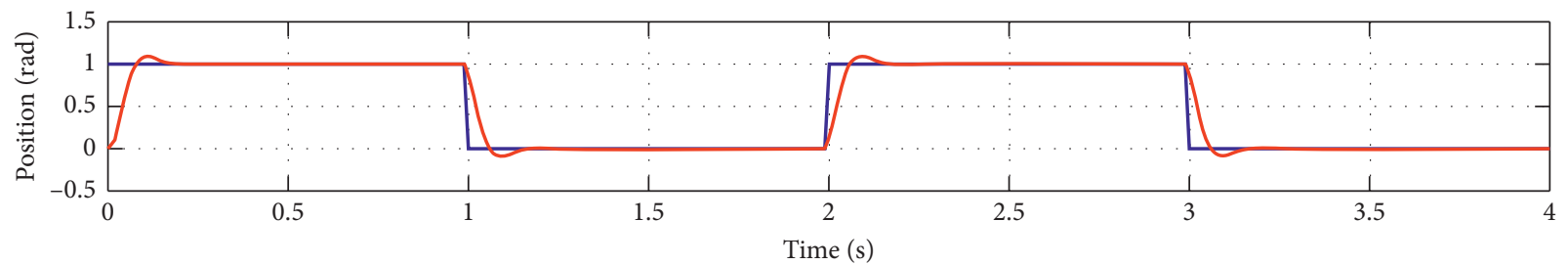

(a)

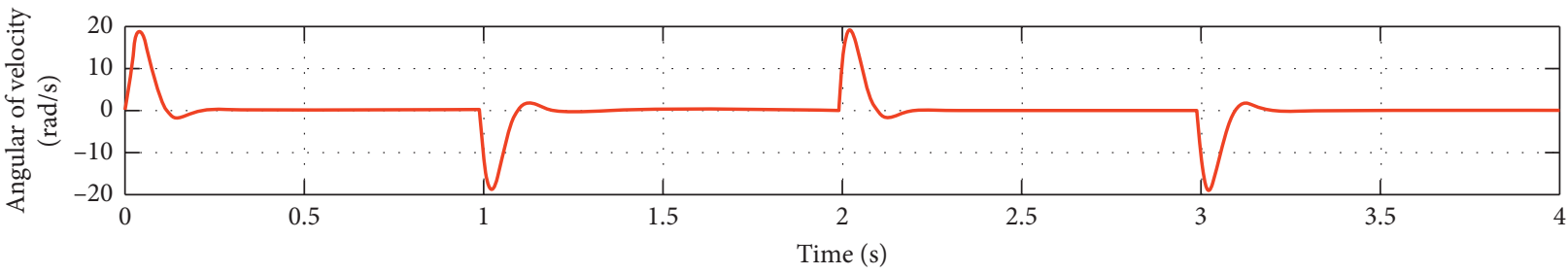

(b)

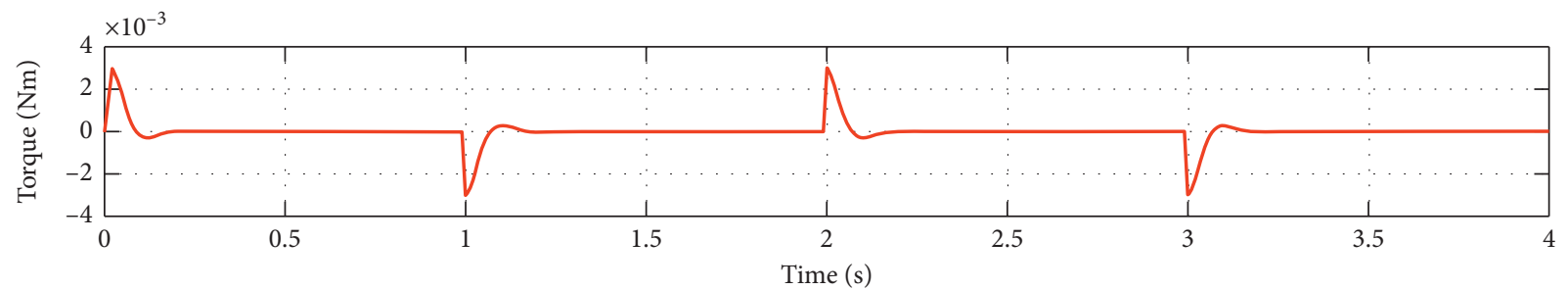

(c)

FIGURE 3: Simulation performance of a conventional PID controller without uncertainties in the driving mechanism (blue line: reference signal; red line: feedback signal).

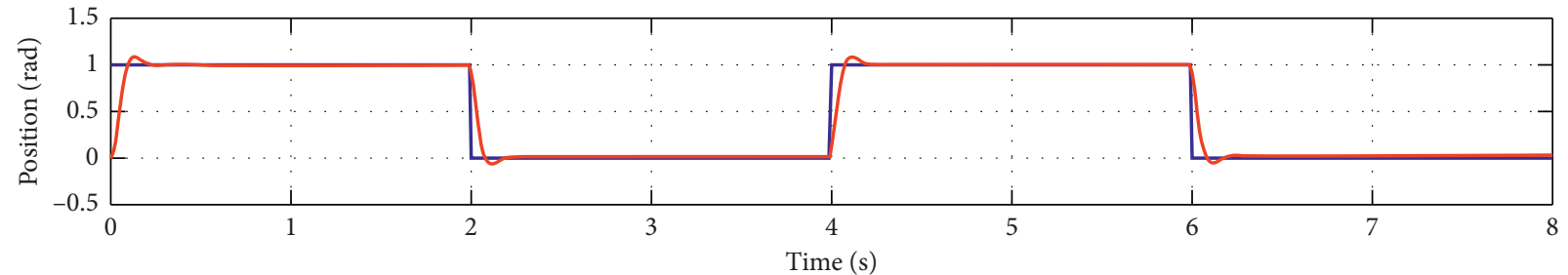

(a)

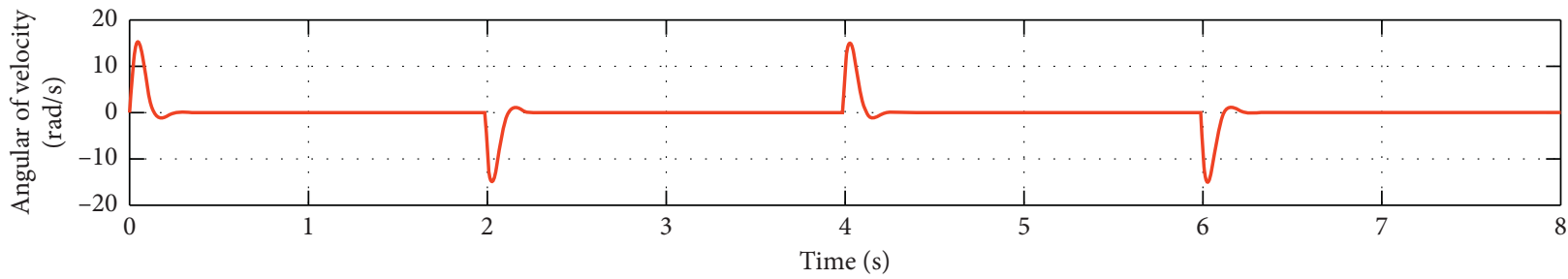

(b)

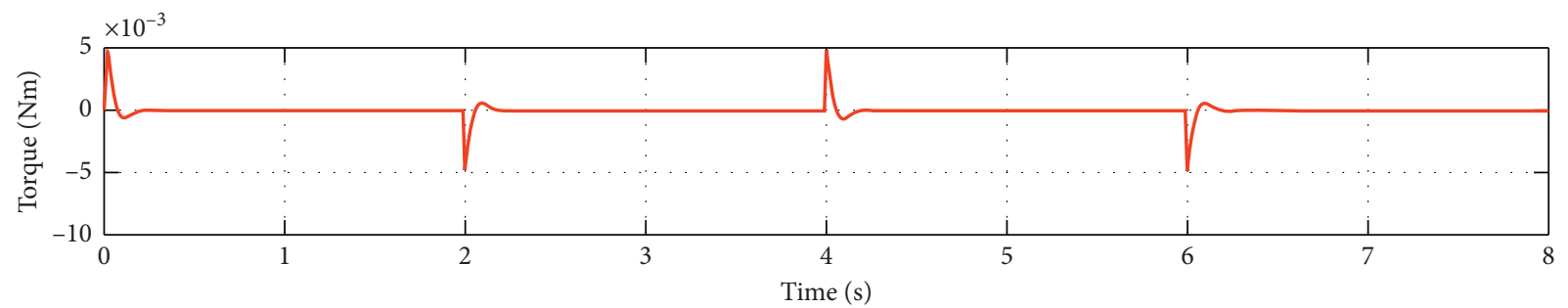

(c)

FIGURE 4: Simulation performance of the proposed controller without uncertainties in the driving mechanism (blue line: reference signal; red line: feedback signal). 


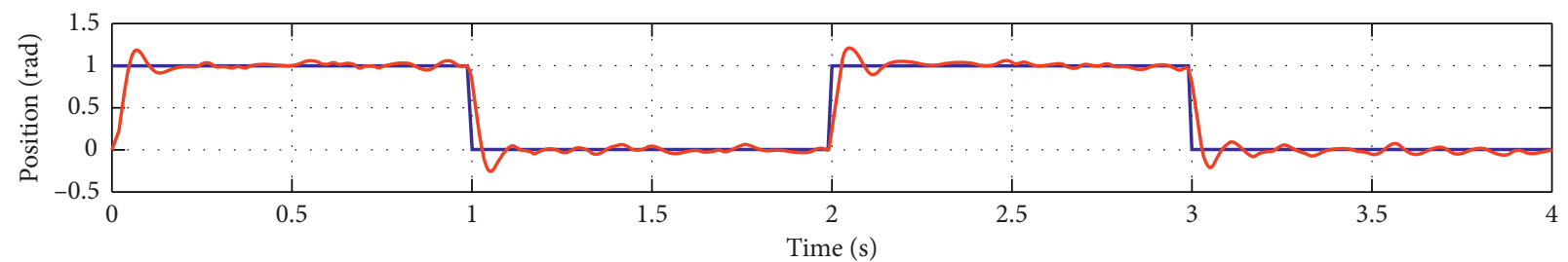

(a)

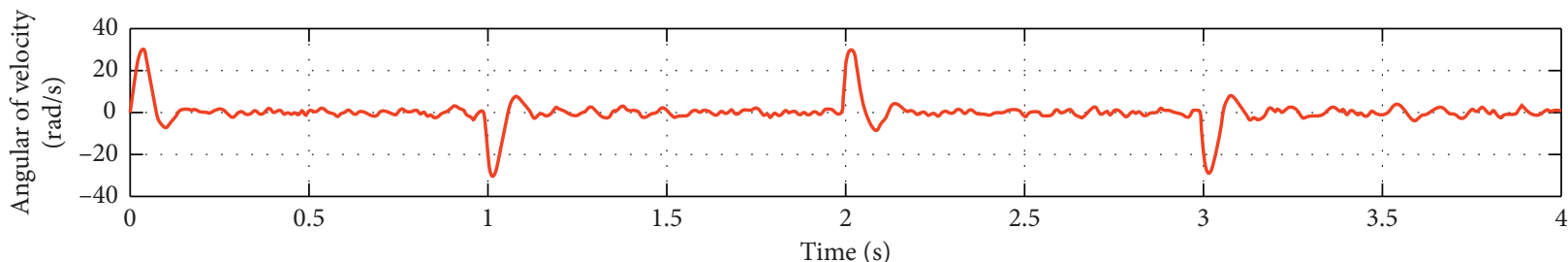

(b)

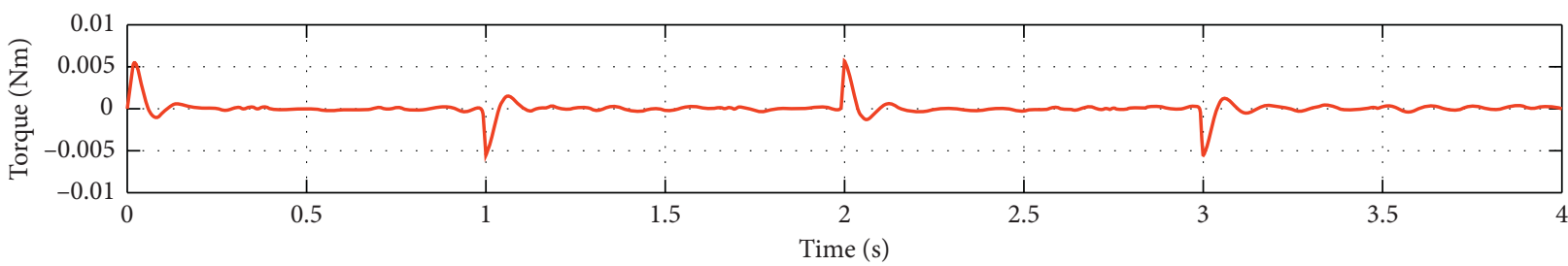

(c)

FIGURE 5: Simulation performance of a conventional PID controller with uncertainties in the driving mechanism (blue line: reference signal; red line: feedback signal).

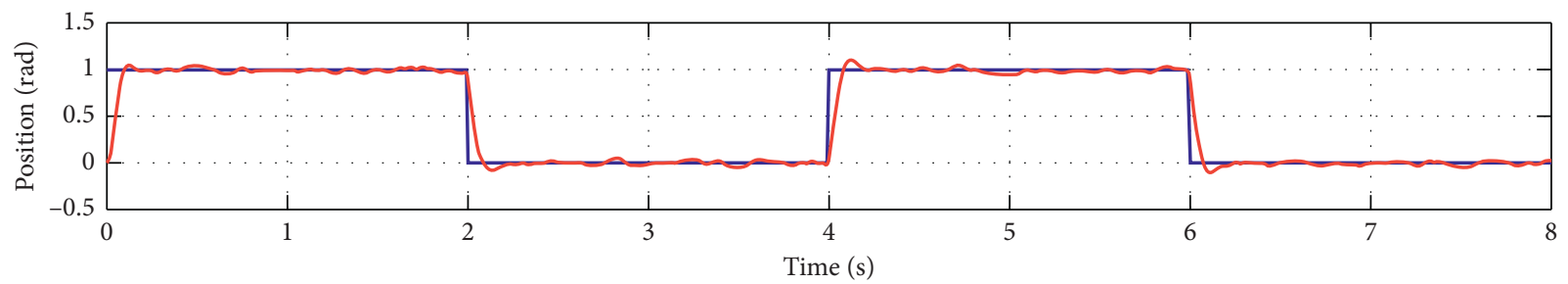

(a)

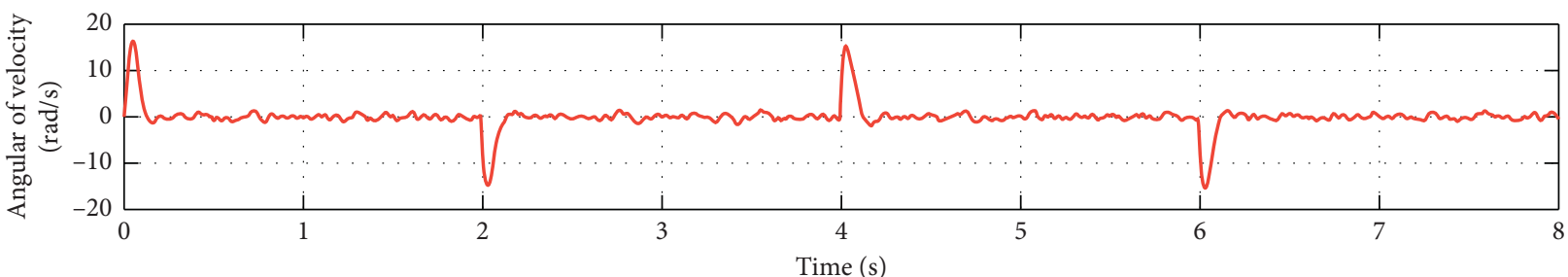

(b)

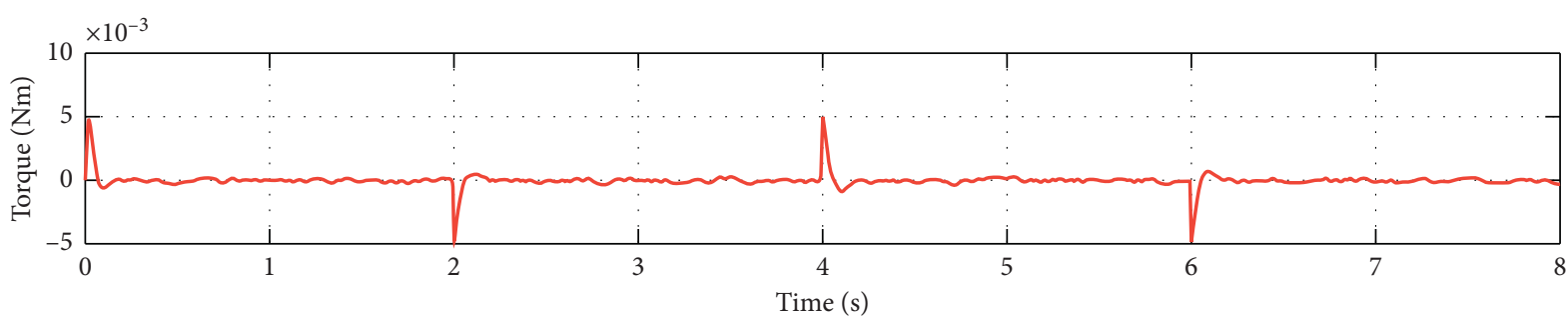

(c)

FIGURE 6: Simulation performance of the proposed controller with uncertainties in the driving mechanism (blue line: reference signal; red line: feedback signal). 


\section{Results of Experimental Verification}

The experimental hardware shown in Figure 7 comprises a servo motion system (embedded motion controller, servo driver, servo motor, ball-screw mechanism, load and linear scale ruler). The system software includes a monitoring program (Windows-based operating system) and a real-time firmware (C-based programming language). In this subsection, the robust properties of controllers whose control algorithms are implemented in firmware are investigated. The host computer plays a principal role in the interaction with operators, while the embedded motion manages data flow at the board level. To prevent data loss, on each side, a circular buffer was programmed to store and obtain data simultaneously. As a result, data transmission was ensured at high speed and with high reliability. The servo driver was set up in position control mode and the velocity was found by backward difference of the position signal. The parameters of the controllers and the boundary conditions were the same as in the simulation tests.

A micro-processor-based controller was developed to verify the proposed control approach. In order to mimic practical scenarios, all design processes comprising electronic components, schematics, and PCB routing followed industrial standards. Figure 8 illustrates the physical connection from the host side to the servo side. In the Windows environment, a C-based thread in motion library, which helps to maintain continuous data transmission, executed read and write commands continuously. User access on the API functioned to monitor all system states in the graph of the control screen. The external link between the computer server and motion controller was by USB-to-serial wiring, while the internal communication in the embedded board used SPI and I2C protocol. Depending on the functional purpose, the design of the board is divided into three parts: CPU module, base module, and GPIO module. The central processor unit ARM Cortex M4 [57] was placed as a CPU module. It handles data to the host computer through a USB bridge IC and to other segments with the aid of a communication bus. This ARM chip is a highly efficient embedded processor, up to $80 \mathrm{MHz}$, with low power, a large memory with $256 \mathrm{kB}$ Flash, $32 \mathrm{kB}$ SRAM and $2 \mathrm{kB}$ EEPROM; is of low cost; and has floating-point support. As the base module, the design was integrated with a PCL-6045 [58] chip from NIPPON PULSE, which is a high-performance motion IC with advanced control motion features, supported by four axes, with a linear/circular/continuous interpolation and trapezoidal/S-curve profile. Furthermore, some isolated circuits were used to protect the controller from backwardcurrent. Two differential signals went from pulse port and dir port to the servo interface. Synchronous in/out and manual pulse control were employed to match the input and output, respectively, and to generate the desired number of pulses. In a general purpose input/output module, a series of LEDs as well as connector pins designate the level of signals.

At the firmware level, the programming environment was IAR workbench, which uses a $\mathrm{C} / \mathrm{C}++$ compiler, assembler, and linker. The architecture of the embedded software is described in Figure 9. The controller constantly

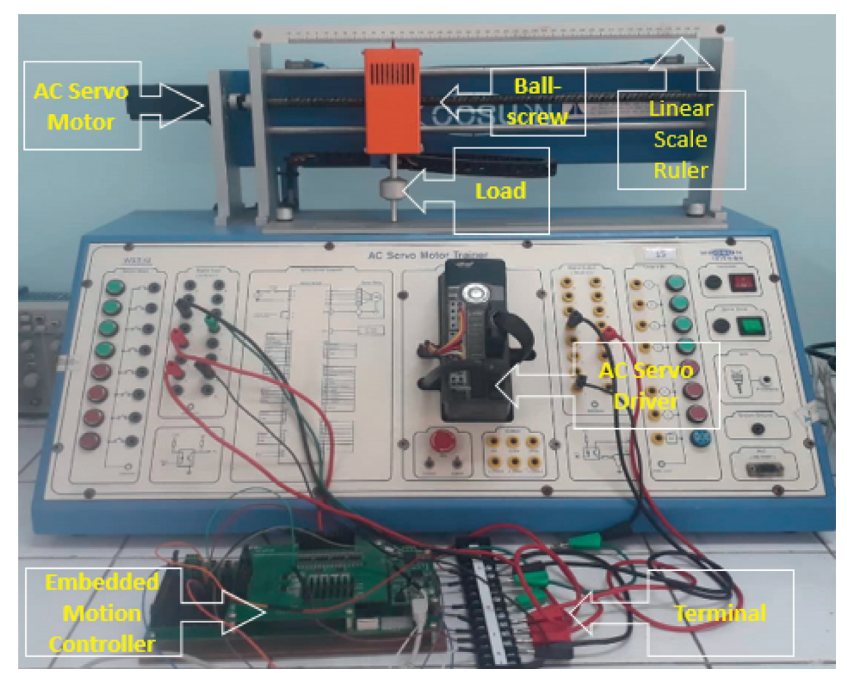

Figure 7: The experimental AC servo control system (embedded controller, AC servo driver, AC servo motor, ball-screw mechanism, load and linear scale ruler).

runs in the main control loop to manage data exchange, read information from sensors, check the servo status, and hold in/out. In one servo cycle, the motion profile generates a point-to-point trajectory plan from the polynomial function. The sampling interval is $0.25 \mathrm{~ms}$, allowing enough time execution for the planner to accomplish a step of motion. Then, an interrupt signal is acknowledged by the host if the movement is complete or if any error takes place. To apply it in more areas, more functions and utilities can be realized in the controller. Henceforth, a manager subprogram administers motion commands, sensing commands, and multitasking commands.

The servo platform would unavoidably be subject to parameter deviations, nonlinear forces, or troubles in highfrequency dynamics, so a theoretical analysis alone is not convincing. Therefore, real-world tests were performed with the apparatus in Figure 7. In these cases, a point-to-point reference technique that is well known in numerous positioning motion systems was engaged to prove the control performance of the controllers under various circumstances. Figures 10 and 11 present the output results of the real investigations with the PID controller and the proposed controller with uncertainties, respectively. It can be seen that an oscillation exists in the measured signal with nonlinear uncertainties in the PID scheme. An enhanced outcome with a better performance was achieved with the proposed scheme. When the robust control law based on the PFSMC strategy is added to the servo system, it can attenuate the effect of uncertainties. The timing motion generation in the proposed control approach is shorter because it does not rely on vibrationless movement. Consequently, the superior specifications in terms of rapid reaction, high positioning precision, less vibration, and increased robustness were validated in the laboratory test.

In terms of practical application, there are several relations between fuzzy control and tuning coefficients. The effect of the fuzzy control component is larger when there is 
TABLE 2: Comparative simulation results among controllers.

\begin{tabular}{lccc}
\hline Rule no. & Overshoot (\%) & Rising time (s) & RMS tracking error (rad) \\
\hline Without uncertainties & & & 0.14 \\
PID controller & 10.1 & 0.05 & 0.17 \\
PFSMC controller & 7.3 & 0.08 & \\
\hline With uncertainties & & 0.21 & 0.30 \\
PID controller & 15.2 & 0.13 & 0.16 \\
PFSMC controller & 9.5 &
\end{tabular}

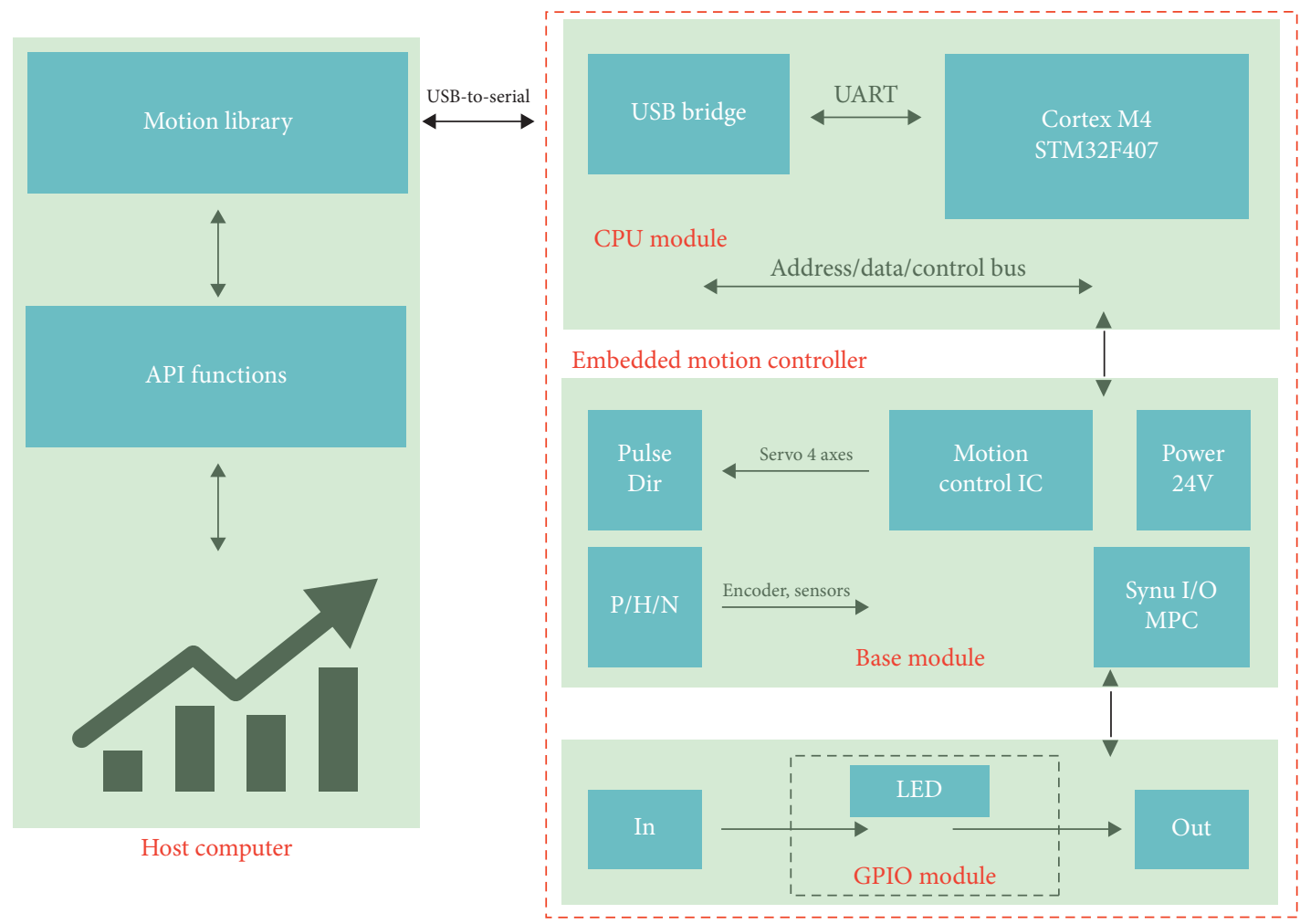

FIGURE 8: The flowchart of overall communication in the AC servo control system.

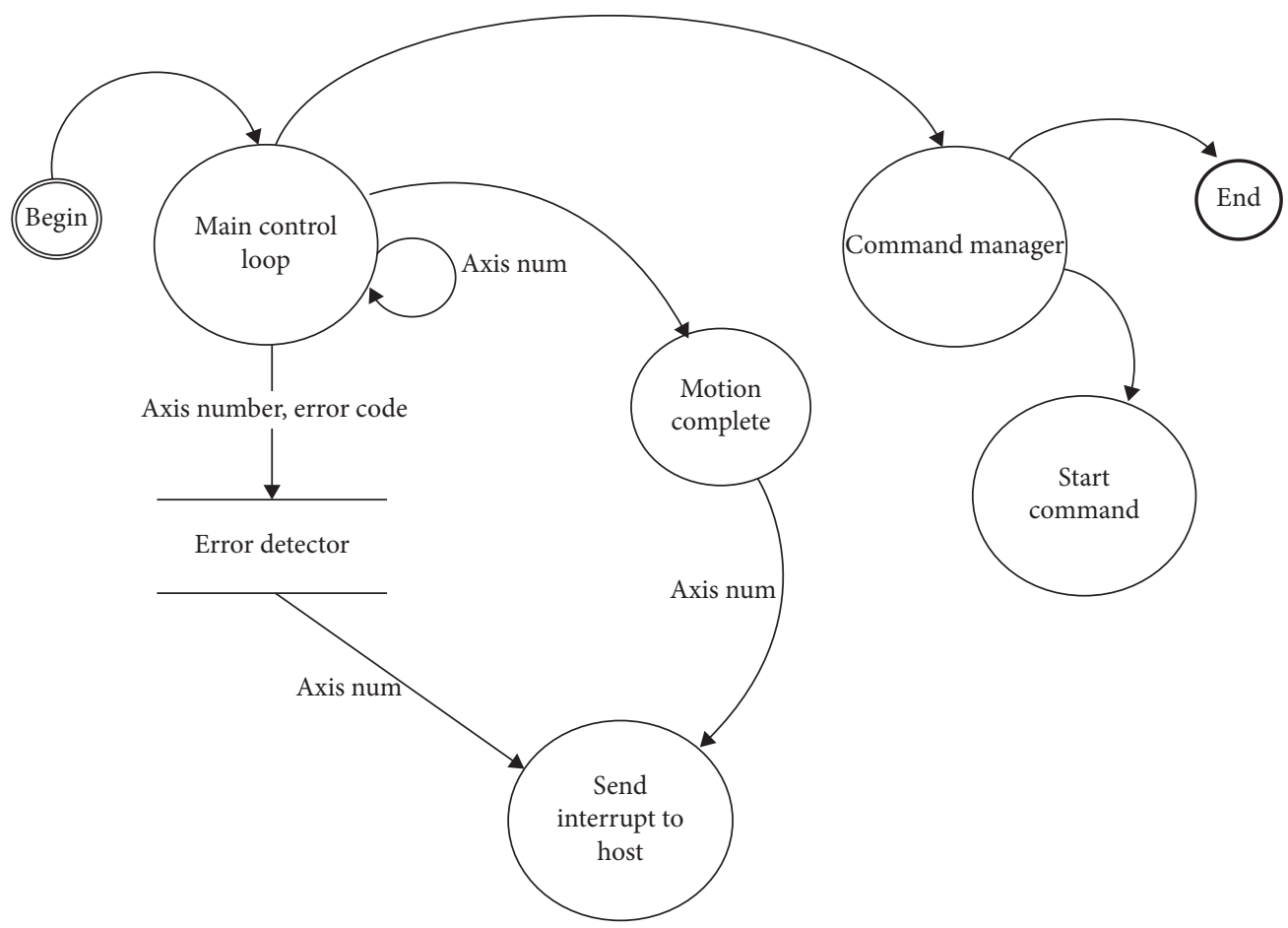

FIGURE 9: The flowchart of overall communication in the AC servo control system. 


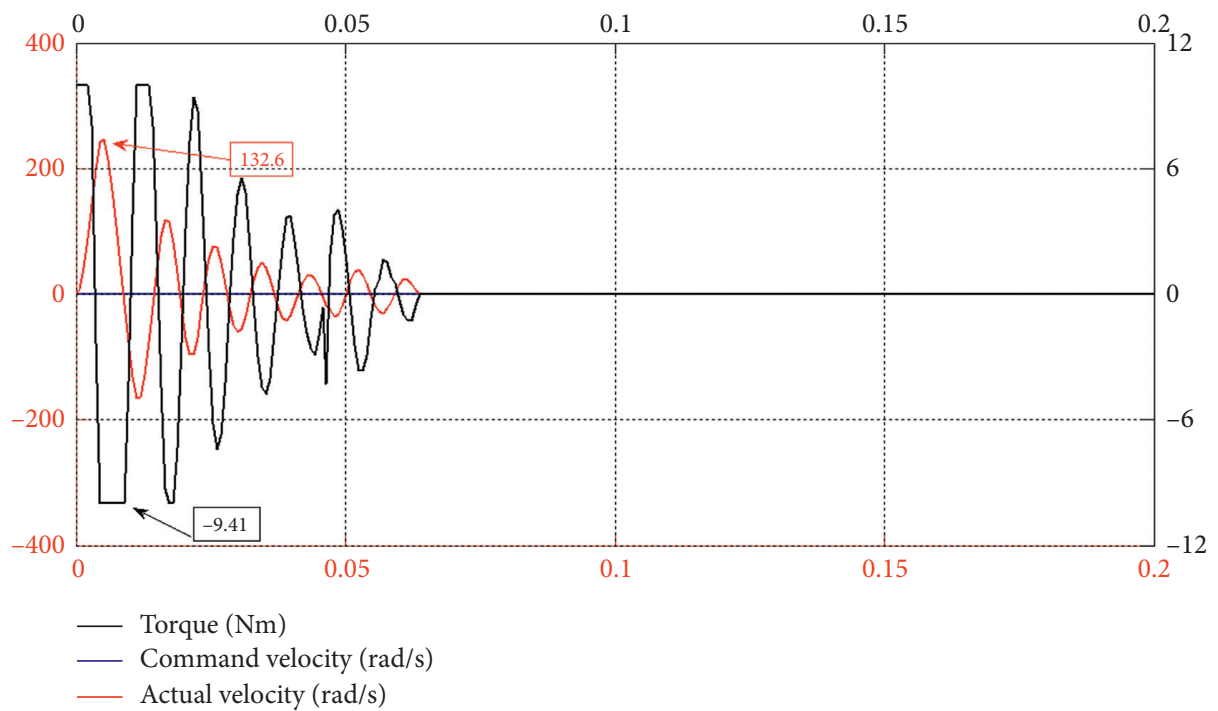

FIGURE 10: Experimental performance of the PID controller in the presence of uncertainties in practical servo system.

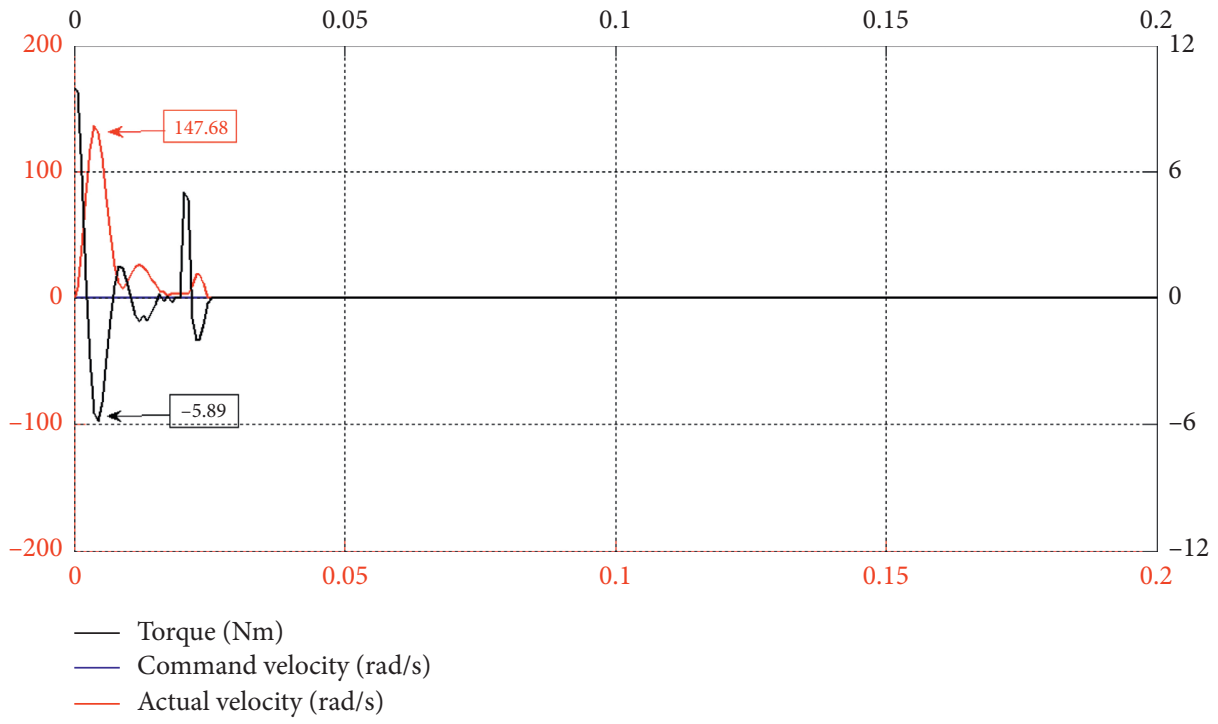

FIGURE 11: Experimental performance of the proposed controller in the presence of uncertainties in practical servo system.

an increase in the sliding function magnitude $\left|\Delta s_{i}\right|$. Nevertheless, their signs are contradictory. When tuning, the fuzzy control has the same effect on the overall control signals. It is rather sensitive to both the sliding function $s_{i}$ and sliding vector $s$ in the case that $\mu_{\Delta s_{i}}, \mu_{s_{i}}$, or $\mu_{\varphi_{i}}$ are subject to the magnitude of the sliding functions, whilst $\gamma_{i}^{m}$ or $u_{\mathrm{Fz}}$ depend on the saturation condition of the control input signal.

\section{Conclusions}

In this paper, a novel control application for AC servo system was investigated. The contributions can be fully described as follows:

(i) With the SMC approach, robustness and asymptotical stability based on the Lyapunov theory could be achieved. Combined with fuzzy principle, the tracking performance is expected to attenuate disturbances and decrease the sensitivity to uncertainties with a boundary threshold. The fuzzy-like architecture not only promises simple programming but also ensures real-time output.

(ii) An AC servo model was built on a computer to simulate the system response. From mathematical equations, the dynamic characteristics and theoretical assumptions were perfectly performed to overcome the control problems.

(iii) An industrial motion hardware framework was established to approve the proposed scheme. The printed circuit board of the motion controller was designed to embed the control algorithm using $\mathrm{C}$ code. The peripheral instruments, including a servo 
driver, servo motor, and mechanical actuators, were obtained from popular industry partners.

(iv) The extensive comparisons of simulations and experiments clearly demonstrated the effectiveness of the proposed controller and validated that an improved robustness and a superior disturbance rejection result can be obtained by applying the SMC approach into the pseudo fuzzy scheme.

Using the proposed controller, the servo system could explicitly perform chattering-reduced effects with high insensitivity to load variations and unknown system parameters. The tracking error performance and the end-point path planning validated the robustness of the system employing our controller against external disturbances and parameter uncertainty. It is well acknowledged that a tradeoff occurs between robustness and chattering. The application of our technique to the field of industrial control requires further study. A powerful microprocessor should also be considered because of computation burdens. For multiaxis motion control, the effects on tracking performance and synchronization need to be verified. Last but not least, several optimal algorithms could be innovated to choose the proper gains of the controller.

\section{Data Availability}

No data were used to support this study.

\section{Conflicts of Interest}

The authors declare no conflicts of interest.

\section{Acknowledgments}

This research was funded by Japan International Cooperation Agency Project for Asean University Network/Southeast Asia Engineering Education Development Network (JICA Project for AUN/SEED-Net) in the framework of Special Program for Research against COVID-19 Japanese Fiscal Year 2020-2021 (SPRAC) with the support of time and facilities from Ho Chi Minh City University of Technology (HCMUT), VNU-HCM, for this study.

\section{References}

[1] W. Chen, D. Huo, Y. Shi, and J. M. Hale, "State-of-the-art review on vibration-assisted milling: principle, system design and application," International Journal of Advanced Manufacturing Technology, vol. 97, no. 5-8, pp. 2033-2049, 2018.

[2] A. Maghareh, C. E. Silva, and S. J. Dyke, "Parametric model of servo-hydraulic actuator coupled with a nonlinear system: experimental validation," Mechanical Systems and Signal Processing, vol. 104, pp. 663-672, 2018.

[3] C. Wang, J. Pan, Y. Hong, and Y. Liu, "Design mechanism of sampling frequency on mechanical parameter identification in a two-mass servo drive system," in Proceedings of the 22nd International Conference on Electrical Machines and Systems, pp. 663-672, Harbin, China, March 2019.
[4] L. Cao, A. Downey, S. Laflamme, D. Taylor, and J. Ricles, "Variable friction device for structural control based on duoservo vehicle brake: modeling and experimental validation," Journal of Sound and Vibration, vol. 348, pp. 41-56, 2015.

[5] M. Myint, K. Yonemori, A. Yanou, M. Minami, and S. Ishiyama, "Visual-servo-based autonomous docking system for underwater vehicle using dual-eyes camera 3D-pose tracking," in Proceedings of the IEEE/SICE International Symposium on System Integration, pp. 989-994, Nagoya, Japan, December 2015.

[6] D. Saravanakumar, B. Mohan, and T. Muthuramalingam, "A review on recent research trends in servo pneumatic positioning systems," Precision Engineering, vol. 49, pp. 481-492, 2017.

[7] Q. P. Ha, Q. H. Nguyen, D. C. Rye, and H. F. Durrant-Whyte, "Fuzzy sliding-mode controllers with applications," IEEE Transactions on Industrial Electronics, vol. 48, no. 1, pp. 38-46, 2001.

[8] Q. Feng, L. Wang, L. Su, W. Huang, and B. Hu, "Servo control algorithm of handling manipulator based on disturbing observer," Acta Technica, vol. 62, no. 2, pp. 153-164, 2017.

[9] K. Matsuse and D. Matsuhashi, "New technical trends on adjustable speed AC motor drives," Chinese Journal of Electrical Engineering, vol. 3, no. 1, pp. 1-9, 2017.

[10] A. Visioli, "Research trends for PID controller," Acta Polytechnica, vol. 52, no. 5, pp. 144-150, 2012

[11] H. Q. T. Ngo and W. H. Kim, "Implementation of fuzzy selftuning PID and feed-forward design for high-performance motion control system," International Journal of Fuzzy Logic and Intelligent Systems, vol. 14, no. 2, pp. 136-144, 2014.

[12] J. M. S. Ribeiro, M. F. Santos, M. J. Carmo, and M. F. Silva, "Comparison of PID controller tuning methods: analytical/ classical techniques versus optimization algorithms," in Proceedings of the 18th International Carpathian Control Conference, pp. 533-538, Sinaia, Romania, May 2017.

[13] C. Chen, C. Zhang, T. Hu, H. Ni, and W. Luo, "Model-assisted extended state observer-based computed torque control for trajectory tracking of uncertain robotic manipulator systems," International Journal of Advanced Robotic Systems, vol. 5, no. $5,2018$.

[14] X. Wang and B. Hou, "Trajectory tracking control of a 2-DOF manipulator using computed torque control combined with an implicit lyapunov function method," Journal of Mechanical Science and Technology, vol. 32, no. 6, pp. 2803-2816, 2018.

[15] B. Jaemin, J. Maolin, and H. Soohee, "A new adaptive slidingmode control scheme for application to robot manipulators," IEEE Transactions on Industrial Electronics, vol. 63, no. 6, pp. 3628-3637, 2016.

[16] H. V. A. Truong, D. T. Tran, X. D. To, K. K. Ahn, and M. Jin, "Adaptive fuzzy backstepping sliding mode control for a 3DOF hydraulic manipulator with nonlinear disturbance observer for large payload variation," Applied Sciences, vol. 9, no. 16, p. 3290, 2019.

[17] D. Sun, F. Naghdy, and H. Du, "Neural network-based passivity control of teleoperation system under time-varying delays," IEEE Transactions on Cybernetics, vol. 47, no. 7, pp. $1666-1680$.

[18] C. Hu, Z. Yudong, and J. M. Lee, "LQR control for a mobile manipulator using COG feedback," in Proceedings of the IEEE International Conference on Advanced Intelligent Mechatronics, pp. 921-924, Busan, Korea, July 2015.

[19] S. Odhano, P. Pescetto, H. A. A. Awan, M. Hinkkanen, G. Pellegrino, and R. Bojoi, "Parameter identification and selfcommissioning in AC motor drives: a technology status 
review," IEEE Transactions on Power Electronics, vol. 34, no. 4, 2019.

[20] M. Gomez-Gonzalez, F. Jurado, and I. Pérez, "Shuffled frogleaping algorithm for parameter estimation of a double-cage asynchronous machine," IET Electric Power Applications, vol. 6, no. 8, pp. 484-490, 2012.

[21] K. Liu, J. Feng, S. Guo, L. Xiao, and Z. Q. Zhu, "Improved position offset based parameter determination of permanent magnet synchronous machines under different load conditions," IET Electric Power Applications, vol. 11, no. 4, pp. 603-612, 2017.

[22] S. A. Odhano, A. Cavagnino, R. Bojoi, and A. Tenconi, "Induction motor magnetizing characteristic identification at standstill with single-phase tests conducted through the inverter," in Proceedings of the IEEE International Electric Machines and Drives Conference, pp. 960-966, Coeur d'Alene, ID, USA, May 2015.

[23] S.-H. Lee, A. Yoo, H.-J. Lee, Y.-D. Yoon, and B.-M. Han, "Identification of induction motor parameters at standstill based on integral calculation," IEEE Transactions on Industry Applications, vol. 53, no. 3, pp. 2130-2139, 2017.

[24] S. A. Odhano, P. Giangrande, R. I. Bojoi, and C. Gerada, "Selfcommissioning of interior permanent- magnet synchronous motor drives with high-frequency current injection," IEEE Transactions on Industry Applications, vol. 50, no. 5, pp. 3295-3303, 2014.

[25] S. Sakunthala, R. Kiranmayi, and P. N. Mandadi, "A study on industrial motor drives: comparison and applications of PMSM and BLDC motor drives," in Proceedings of the 2017 International Conference on Energy, Communication, Data Analytics and Soft Computing (ICECDS), pp. 537-540, IEEE, Chennai, India, August 2017.

[26] R. J. E. Merry, N. C. T. De Kleijn, M. J. G. Van De Molengraft, and M. Steinbuch, "Using a walking piezo actuator to drive and control a high-precision stage," IEEE/ASME Transactions on Mechatronics, vol. 14, no. 1, pp. 21-31, 2009.

[27] C. Zhao, Q. Zhang, W. Zhang et al., "Hybrid piezo/triboelectric nanogenerator for highly efficient and stable rotation energy harvesting," Nano Energy, vol. 57, pp. 440-449, 2019.

[28] X. Li, P. Ci, G. Liu, and S. Dong, "A two-layer linear piezoelectric micromotor," IEEE Transactions on Ultrasonics, Ferroelectrics, and Frequency Control, vol. 62, no. 3, pp. 405-411, 2015.

[29] J. F. Carneiro and F. G. de Almeida, "Friction characteristics and servo control of a linear peristaltic actuator," The International Journal of Advanced Manufacturing Technology, vol. 96, no. 5, pp. 2117-2126, 2018.

[30] Y. Matsui, T. Akagi, and S. Dohta, "Development of low-cost wire type linear potentiometer for flexible spherical actuator," in Proceedings of the 2016 IEEE International Conference on Advanced Intelligent Mechatronics (AIM), pp. 1017-1021, IEEE, Banff, Canada, July 2016.

[31] J. Talla, V. Q. Leu, V. Šmídl, and Z. Peroutka, "Adaptive speed control of induction motor drive with inaccurate model," IEEE Transactions on Industrial Electronics, vol. 65, no. 11, pp. 8532-8542, 2018.

[32] F. F. M. El-Sousy, "Adaptive dynamic sliding-mode control system using recurrent RBFN for high-performance induction motor servo drive," IEEE Transactions on Industrial Informatics, vol. 9, no. 4, pp. 1922-1936, 2013.

[33] H. Q. T. Ngo, T. P. Nguyen, T. S. Le, V. N. S. Huynh, and H. A. M. Tran, "Experimental design of PC-based servo system," in Proceedings of the 2017 International Conference on System Science and Engineering (ICSSE), pp. 733-738, IEEE, Ho Chi Minh City, Vietnam, July 2017.

[34] Y. Yaolong Tan, J. Jie Chang, and H. Hualin Tan, "Adaptive backstepping control and friction compensation for AC servo with inertia and load uncertainties," IEEE Transactions on Industrial Electronics, vol. 50, no. 5, pp. 944-952, 2003.

[35] R.-H. Zhang, Z.-C. He, H.-W. Wang, F. You, and K.-N. Li, "Study on self-tuning tyre friction control for developing main-servo loop integrated chassis control system," IEEE Access, vol. 5, pp. 6649-6660, 2017.

[36] H. A. Mintsa, R. Venugopal, J. P. Kenne, and C. Belleau, "Feedback linearization-based position control of an electrohydraulic servo system with supply pressure uncertainty," IEEE Transactions on Control Systems Technology, vol. 20, no. 4, pp. 1092-1099, 2011.

[37] V. T. Vu, O. Sename, L. Dugard, and P. Gáspár, "Enhancing roll stability of heavy vehicle by LQR active anti-roll bar control using electronic servo-valve hydraulic actuators," Vehicle System Dynamics, vol. 55, no. 9, pp. 1405-1429, 2017.

[38] S. Wang, J. Na, and X. Ren, "RISE-based asymptotic prescribed performance tracking control of nonlinear servo mechanisms," IEEE Transactions on Systems, Man, and Cybernetics: Systems, vol. 48, no. 12, pp. 2359-2370, 2017.

[39] C. Kaddissi, J. P. Kenne, and M. Saad, "Indirect adaptive control of an electrohydraulic servo system based on nonlinear backstepping," IEEE/ASME Transactions on Mechatronics, vol. 16, no. 6, pp. 1171-1177, 2010.

[40] R.-E. Precup, R.-C. David, E. M. Petriu, S. Preitl, and M.-B. Radac, "Novel adaptive gravitational search algorithm for fuzzy controlled servo systems," IEEE Transactions on Industrial Informatics, vol. 8, no. 4, pp. 791-800, 2012.

[41] J. Na, S. Wang, Y. J. Liu, Y. Huang, and X. Ren, "Finite-time convergence adaptive neural network control for nonlinear servo systems," IEEE Transactions on Cybernetics, vol. 50, no. 6, pp. 2568-2579, 2019.

[42] L. Zhao, B. Zhang, H. Yang, and Y. Wang, "Finite-time tracking control for pneumatic servo system via extended state observer," IET Control Theory \& Applications, vol. 11, no. 16, pp. 2808-2816, 2017.

[43] K. H. Rew and K. S. Kim, "A closed-form solution to asymmetric motion profile allowing acceleration manipulation," IEEE Transactions on Industrial Electronics, vol. 57, no. 7, pp. 2499-2506, 2009.

[44] Z. Rymansaib, P. Iravani, and M. N. Sahinkaya, "Exponential trajectory generation for point to point motions," in Proceedings of the 2013 IEEE/ASME International Conference on Advanced Intelligent Mechatronics, pp. 906-911, IEEE, Ho Chi Minh City, Vietnam, July 2013.

[45] S. Perumaal and N. Jawahar, "Synchronized trigonometric S-curve trajectory for jerk-bounded time-optimal pick and place operation," International Journal of Robotics and Automation, vol. 27, no. 4, p. 385, 2012.

[46] H. Li, "A jerk-constrained asymmetric motion profile for high-speed motion stages to reduce residual vibration," International Journal of Computer Applications in Technology, vol. 53, no. 2, pp. 149-156, 2016.

[47] D. Lee and C.-W. Ha, "Optimization process for polynomial motion profiles to achieve fast movement with low vibration," IEEE Transactions on Control Systems Technology, vol. 28, no. 5, pp. 1892-1901, 2020.

[48] A. Amthor, J. Werner, A. Lorenz, S. Zschaeck, and C. Ament, "Asymmetric motion profile planning for nanopositioning and nanomeasuring machines," Proceedings of the Institution 
of Mechanical Engineers, Part I: Journal of Systems and Control Engineering, vol. 224, no. 1, pp. 79-92, 2010.

[49] Y. Fang, J. Hu, W. Liu, Q. Shao, J. Qi, and Y. Peng, "Smooth and time-optimal S-curve trajectory planning for automated robots and machines," Mechanism and Machine Theory, vol. 137, pp. 127-153, 2019.

[50] L. Biagiotti and C. Melchiorri, “Trajectory generation via FIR filters: a procedure for time-optimization under kinematic and frequency constraints," Control Engineering Practice, vol. 87, pp. 43-58, 2019.

[51] L. Moriello, L. Biagiotti, and C. Melchiorri, "Multidimensional trajectories generation with vibration suppression capabilities: the role of exponential B-splines " * " * this activity has been supported by the University of Modena and Reggio Emilia with the "FAR 2015" project," IFAC-PapersOnLine, vol. 50, no. 1, pp. 6054-6059, 2017.

[52] Y. Fang, J. Qi, J. Hu, W. Wang, and Y. Peng, “An approach for jerk-continuous trajectory generation of robotic manipulators with kinematical constraints," Mechanism and Machine Theory, vol. 153, Article ID 103957, 2020.

[53] S. A. Odhano, P. Pescetto, H. A. A. Awan, M. Hinkkanen, G. Pellegrino, and R. Bojoi, "Parameter identification and selfcommissioning in AC motor drives: a technology status review," IEEE Transactions on Power Electronics, vol. 34, no. 4, pp. 3603-3614, 2018.

[54] N. Bedetti, S. Calligaro, and R. Petrella, "Stand-still selfidentification of flux characteristics for synchronous reluctance machines using novel saturation approximating function and multiple linear regression," IEEE Transactions on Industry Applications, vol. 52, no. 4, pp. 3083-3092, 2016.

[55] S. M. Yang and K. W. Lin, "Automatic control loop tuning for permanent-magnet AC servo motor drives," IEEE Transactions on Industrial Electronics, vol. 63, no. 3, pp. 1499-1506, 2015.

[56] Higen Electric Drive, CN Series Servo System User's Manual, Higen Electric Drive, Changwon-si, South Korea, 2010.

[57] STM32F4 Series, 2020. https://www.st.com/en/ microcontrollers-microprocessors/stm32f4-series.html.

[58] PCL6045 user's manual, 2020. https://www.pulsemotor.com/ global/. 